\title{
REVISTAS LATINOAMERICANAS DE EDUCACIÓN INDEXADAS EN SCOPUS
}

\author{
LATIN AMERICAN JOURNALS OF EDUCATION \\ INDEXED IN SCOPUS
}

Wendy Arhuis Inca ${ }^{1}$ Julio Cjuno²

\section{Sr. Editor}

La labor de los educadores es guiar, formar y orientar a los estudiantes, preparándolos para la vida; es decir, ayudarles a desarrollar actitudes para la toma de decisiones, y crear así sociedades sostenibles ${ }^{1}$. Un educador que investiga, tiene la capacidad y el pensamiento crítico para proponer reformas e innovación en educación y pedagogía, buscando mejorar la calidad en ese ámbito, asimismo, es capaz de transmitir estas capacidades a sus estudiantes ${ }^{2}$, por ello, la presencia de cada vez más cantidad de educadores investigadores es necesario para el desarrollo de los países.

Las revistas científicas son canales a través de los cuales se realizan publicaciones periódicas ya sea por medios electrónicos o impresos ${ }^{3}$. Estas publicaciones contienen resultados de investigaciones que se desean transmitir a un público lector diverso, como: investigadores, académicos, estudiantes, gobernantes y tomadores de decisiones, con la finalidad de que aquellos resultados sean utilizados como base para generar cambios, mejoras o políticas que beneficien a la sociedad. ${ }^{4}$

1 Licenciada en Educación. Instituto de Investigación, Universidad Católica Los Ángeles de Chimbote, Chimbote, Perú. Email: warhuisi@uladech.edu.pe

2 Licenciado en Psicología. Instituto de Investigación, Universidad Católica Los Ángeles de Chimbote, Chimbote, Perú. 
Scopus es una base de datos de alto impacto a nivel internacional, que contiene literatura científica de todas las áreas del conocimiento; además, cuenta con herramientas útiles que permiten explorar, examinar, y visualizar algunos datos concernientes a la literatura científica publicada, como los autores, sus afiliaciones institucionales, los países donde se realizan estas publicaciones y la clasificación por áreas de investigación, que son resumidos y presentados a través de métricas de impacto y tendencias ${ }^{5}$.

La indexación de una revista a Scopus es equivalente una mayor visibilidad, la cual conlleva potencialmente a aumentar el número de lectores, una mayor probabilidad de que alguno de sus artículos sea citado y, por ende, a lograr una mejora en sus métricas de impacto ${ }^{6}$. Para que una revista logre la indexación en Scopus, debe cumplir varios criterios de evaluación; entre ellos, la evaluación de políticas de la revista, sus contenidos, la relevancia, periodicidad, el rigor de la revisión por pares y otros indicadores de calidad científica ${ }^{7}$. En ese sentido, es de interés conocer aquellas revistas latinoamericanas de educación indexadas a Scopus, porque asegura que son revistas que brindarán mayor visibilidad e impacto a las publicaciones de los educadores, siendo de interés dar a conocer dicha información a los investigadores de nuestra región.

En diciembre del 2017 se realizó una búsqueda de revistas latinoamericanas de educación indexadas a Scopus a través del buscador Scimago Journal \& Contry Rank $^{8}$. Encontramos un total de $(\mathrm{N}=23)$ revistas de educación, Brasil es el país con más revistas indexadas a Scopus $(\mathrm{N}=13)$, seguido de México $(\mathrm{N}=5)$, Chile $(\mathrm{N}=3)$, y Colombia $(\mathrm{N}=2)$. El 60,9\% de las revistas pertenecían a universidades, el 8,7\% cobran a autores o lectores, en cuanto al idioma de publicación, el 21,7\% publica en el idioma nativo de su país, mientras que el 60,9\% publica artículos o bien en su idioma nativo o en inglés y el 17,4\% publican paralelamente el mismo artículo en su idioma nativo e inglés (Tabla 1).

Latinoamérica solo tuvo 23 revistas de educación indexadas a Scopus, siendo este un número bajo comparado con Europa, que cuenta con 473 revistas y Norte América, con $345^{\circ}$, Brasil viene a ser el país que lidera con el mayor número de revistas, con mayor número de artículos publicados y es el único país con revistas que publican un artículo en idioma nativo e inglés simultáneamente. Ninguna revista es Q1, solo cuatro países latinoamericanos tienen revistas indexadas a Scopus, la mayoría de revistas pertenecen a universidades y existe una minoría que cobra a autores o lectores, esto podría ser probablemente debido a que las 
revistas son financiadas por universidades y por ello, no requieren de algún tipo de pago por publicar o leer.

En conclusión, existen pocas revistas de educación latinoamericanas indexadas a Scopus, algunas han optado por publicar sus artículos en su idioma nativo e inglés que viene a ser un idioma universal con el afán de aumentar la visibilidad, ninguno es Q1, la mayoría no cobra por publicar o leer y pertenecen a universidades de las que probablemente dependerán económicamente.

Estos hallazgos nos muestran que las revistas de educación de Latino América, tienen un arduo trabajo para consolidar la calidad y mejorar sus métricas de impacto en la base de datos Scopus.

\section{REFERENCIAS}

1. Organización de las Naciones Unidas para la Educación la Ciencia y la Cultura. Educación [Internet]. UNESCO. 2017 [cited 2017 Dec 12]. Available from:

http://www.unesco.org/new/es/education/themes/leading-the-internationalagenda/education-for-sustainable-development/partners/educators/

2. Universidad Central de Venezuela. Escuela de Educación. Revista de pedagogía. Escuela de Educación, Universidad Central de Venezuela;

3. Felquer L. Las revistas científicas: su importancia como instrumento de comunicación de la ciencia. Agentia.Unne.Edu.Ar. 2002.

4. Gatfield RL, Hyde M. An examination of two case studies used in building a decision-making model. Int Educ J. 2005;6(5):555-66.

5. Scopus | The largest database of peer-reviewed literature | Elsevier [Internet]. [cited 2017 Dec 12]. Available from: https://www.elsevier.com/solutions/scopus

6. Universidad de Antioquia. Escuela Interamericana de Bibliotecología. S. Revista interamericana de bibliotecología. Rev Interam Bibl. 2011;34(2).

7. Universidad de Sevilla. Guías de la BUS: Cómo incluir revistas en WOS y Scopus: Criterios de Scopus [Internet]. 2017 [cited 2017 Dec 12]. Available from:

http://guiasbus.us.es/revistas-wos-scopus/criterios_Scopus

8. Scimago Journal \& Country Rank. Journal Rankings of Education [Internet]. 2017 [cited 2017 Dec 13]. Available from:

http://www.scimagojr.com/journalrank.php? category $=3304 \&$ area $=3300 \&$ country $=$ Latin America

9. Scimago Journal \& Country Rank. Journal Rankings of Education [Internet]. 2017 [cited 2017 Dec 13]. Available from:

http://www.scimagojr.com/journalrank $\cdot$ php? category $=3304 \&$ area $=3300 \&$ country $=$ Nort hern America 
Wendy Arhuis Inca, Julio Cjuno

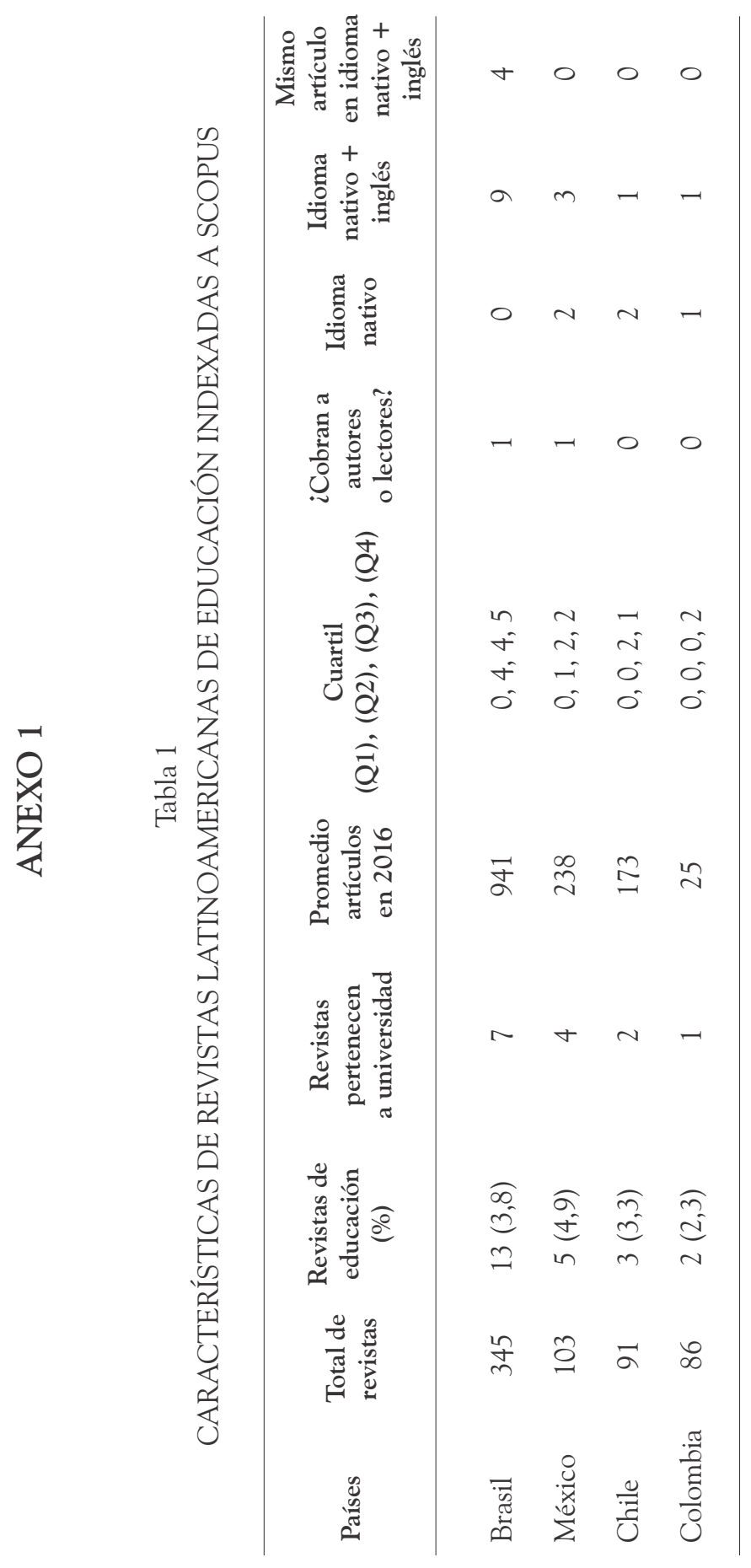

130 | In Crescendo, 2018; 9(1): 127-130 